\title{
Engajamento docente na Pandemia da COVID-19: a influência do suporte
}

\section{organizacional}

\author{
Engagement of university professors in the COVID-19 Pandemic: influence of organizational
}

support

Engagement en el trabajo del profesorado en la Pandemia COVID-19: la influencia del soporte organizacional

\section{Resumo}

Este estudo teve como objetivo identificar a influência do suporte organizacional sobre o engajamento no trabalho durante a Pandemia da COVID-19. Foi realizada uma survey com docentes que atuam no ensino superior da Universidade Federal de Santa Maria. Como técnicas de análise, utilizou-se estatística descritiva, análise fatorial exploratória e regressão. Identifica-se que a adoção do modelo remoto gerou aumento nas demandas de trabalho, em que a maioria dos docentes manteve o foco apesar dos desafios quanto à manutenção de um estado de espírito positivo. O suporte organizacional oferecido pela instituição influência diretamente a capacidade do docente manterse engajado nessa situação atípica e desafiadora. Assim, em contextos emergenciais, como a Pandemia da COVID-19, é fundamental que os gestores universitários disponibilizem mecanismos adequados de forma a proporcionar suporte organizacional necessário para que os docentes se mantenham engajados frente a mudanças drásticas e rápidas no contexto e forma do trabalho docente.

Palavras-chave: Engajamento no trabalho; Suporte organizacional; Pandemia da COVID-19; Ensino.

\begin{abstract}
This study aimed to identify the influence of organizational support on work engagement during the COVID-19 Pandemic. A survey was carried out with professors who work in higher education at the Federal University of Santa Maria. As analysis techniques, descriptive statistics, exploratory factor analysis and regression were used. It is identified that the adoption of the remote model generated an increase in work demands, in which, most professors, kept their focus despite the challenges in maintaining a positive state of mind. The organizational support offered by the institution directly influences the faculty's ability to remain engaged in this atypical and challenging situation. Thus, in emergency contexts, such as the COVID-19 Pandemic, it is essential that university managers provide adequate mechanisms in order to provide the necessary organizational support for teachers to remain engaged in the face of drastic and rapid changes in the context and form of teaching work.
\end{abstract}

Keywords: Work engagement; Organizational support; COVID-19 pandemic; Teaching. 


\begin{abstract}
Resumen
Este estudio tuvo como objetivo identificar la influencia del engagement en el trabajo en durante la Pandemia de COVID-19. Se realizó una encuesta con profesores que trabajan en educación superior en la Universidad Federal de Santa Maria. Como técnicas de análisis se utilizó estadística descriptiva, análisis factorial exploratorio y regresión. Se identifica que la adopción del modelo remoto generó en las demandas de trabajo, en el que, la mayoría de los docentes, mantuvo el foco a pesar de los desafíos en cuanto al mantenimiento de un estado de ánimo positivo. El apoyo organizacional ofrecido por la institución directa influye en la capacidad del maestro para permanecer involucrado en esta situación atípica y desafiante. Por lo tanto, en contextos de emergencia, como la Pandemia de COVID-19, es esencial que los gerentes universitarios proporcionen mecanismos adecuados para proporcionar el apoyo organizativo necesario para que los docentes se mantengan comprometidos frente a los cambios drásticos y rápidos en el contexto y la forma de trabajo docente.
\end{abstract}

Palabras clave: Compromiso en el trabajo; Engagement en el trabajo; Pandemia de COVID-19; Enseñanza.

\title{
1. Introdução
}

Durante a Pandemia da COVID-19, problemas relacionados às condições de trabalho, como a precarização e intensificação, têm-se agravado frente as transformações do ambiente de trabalho e suas demandas (Diab-Bahman \& Al-Enzi, 2020; Tavares, Santos, Diogo, \& Ratten, 2020). Isso ditou a urgência de assumir o desafio de implantar um novo método de ensino, exigindo adaptação de todos os envolvidos (Rodrigues, Franco, \& Silva, 2020), centrando-se, principalmente, nos esforços em torno da aprendizagem e do ensino remoto, visto que houve uma mudança abrupta para aulas online por docentes de Instituições de Ensino Superior (IES) em todo o mundo (Bao, 2020; Adedoyin \& Soykan, 2020; Cardullo, Wang, Burton, \& Dong, 2021; Rapanta, Botturi, Goodyear, Guàrdia, \& Koole, 2020; Zhou, Wu, Zhou, \& Li, 2020).

No entanto, pouco se sabe sobre os impactos da pandemia sobre o trabalho docente (Hoang, 2020), frente a necessidade de modificar o modo de ministrar aulas, adotando novas estratégias de ensino para poder transitar de uma aprendizagem presencial para a remota (Donaldson, 2020). De acordo com Kashyap, Nakra e Arora (2021), as IES estão adotando várias iniciativas para orientar os membros do corpo docente a se manterem engajados no trabalho durante a Pandemia, contudo as investigações e a produção científica e acadêmica sobre o tema são escassas (Rodrigues et al., 2020).

Durante a Pandemia da COVID-19 os ambientes de aprendizagem online continuam a se expandir, exigindo a transição da maioria das IES e universidades para métodos de aprendizagem online (Kamble, Gauba, Desai, \& Golhar, 2021). Reconhecendo a necessidade de pesquisas relacionadas a Pandemia da COVID-19, considera-se a relevância de investigar o efeito do afastamento do trabalho presencial no engajamento no trabalho (EGT), pois conforme Garg, Murphy e Singh (2021), é vantajoso para as organizações se concentrar na criação de um ambiente envolvente, tendo em vista a possibilidade de permitir que os funcionários ajustem seu ambiente de trabalho para atingir com eficiência seus objetivos, estimulando seu crescimento e desenvolvimento pessoal.

Neste contexto, a solução encontrada pelas IES para a manutenção das atividades de ensino foi a utilização do sistema remoto. Na Universidade Federal de Santa Maria (UFSM) foi implantado o Regime de Exercícios Domiciliares Especiais (REDE) (UFSM, 2020a), permitindo que as aulas fossem ministradas através de Tecnologias Educacionais em Rede. A modalidade foi implementada de forma emergencial em função da Pandemia da COVID-19, sendo inicialmente opcional e posteriormente obrigatória, definindo que as ofertas de disciplinas se dariam unicamente neste formato (UFSM, 2020b).

Assim, o modo habitual de desempenhar as atividades inerentes à docência foi significativamente modificado, já que a implantação do REDE impôs a necessidade de ajuste dos planos de ensino, atualização de softwares, metodologias e bibliografias ao formato digital, reconfigurando completamente a rotina de trabalho destes profissionais. Neste contexto, o suporte organizacional e o engajamento no trabalho tornam-se cruciais para que as organizações possam se adaptar às mudanças (Chanana, 2020).

Em vista disso, atenta-se para a necessidade de compreender os efeitos de condições adversas no engajamento e da percepção de suporte organizacional (PSO) (Najeemdeen, Abidemi, Rahmat, \& Bulus, 2018; Erum, Abid, \& Contreras, 2020). 
Tais mudanças decorrentes da Pandemia da COVID-19 podem afetar diretamente o PSO, pois encontram-se relacionadas ao stress, tensão, satisfação sobre o desempenho no trabalho e comprometimento e podem contribuir diretamente para o bem-estar dos indivíduos (Tan, Wang, Qian, \& Lu, 2020).

Conforme Karatepe e Aga (2016) e Tan et al. (2020), o EGT remete ao bem-estar, a motivação dos colaboradores, o quanto sentem-se satisfeitos em relação ao trabalho, envolvendo aspectos como vigor, dedicação e absorção. Caesens e Stinglhamber (2014) e Kose (2016) indicam que o EGT resulta de altos níveis de recursos organizacionais, bem como de recursos pessoais e, portanto, parece razoável que colaboradores engajados invistam recursos adicionais em comportamentos positivos. Dada a relação direta do PSO com o EGT (Tan et al., 2020), fica evidente a necessidade de as organizações entenderem como motivar seus colaboradores a se engajarem com o trabalho (Najeemdeen et al., 2018).

Neste sentido, o estudo aborda a seguinte problemática: "Qual a influência do suporte organizacional sobre o engajamento no trabalho durante a Pandemia da COVID-19?". Para tanto, pretende-se identificar a influência do suporte organizacional sobre o engajamento no trabalho em uma Universidade Pública durante a Pandemia da COVID-19. Reconhecendo que a Pandemia da COVID-19 impõe um contexto sem precedentes, diante do qual não há modelos que orientem e direcionem a atuação profissional (Reimers, Schleicher, Saavedra, \& Tuominen, 2020), busca-se ampliar a base de conhecimento sobre as implicações de um trabalho remoto forçado (Anderson \& Kelliher, 2020). Ademais, pretende-se munir a gestão de informações que lhes permita a montagem de intervenções eficazes em prol de aumentar a eficácia e eficiência das universidades, bem como melhorar o bem-estar dos docentes no trabalho.

\section{Metodologia}

Visando atingir o objetivo proposto de identificar a influência do suporte organizacional sobre o engajamento no trabalho durante a Pandemia da COVID-19, foi realizada uma pesquisa de caráter descritivo com base em uma abordagem quantitativa, a qual tem como premissa obter informações precisas sobre uma população específica, bem como mensurar um evento (Hair et al., 2009). Além disso, quando o objetivo é testar teorias ou predizer comportamento futuro de variáveis, os estudiosos mostram uma preferência por ferramentas quantitativas, baseadas em dados obtidos por questionários (MartínAlcázar, Romero-Fernández, \& Sánchez-Gardey, 2008). Para Cooper e Shindler (2011), os dados quantitativos costumam consistir em respostas de participantes que são codificadas, categorizadas e reduzidas a números para que esses dados possam ser manipulados por análise estatística.

Os estudos descritivos, segundo Cooper e Shindler (2011), servem a uma variedade de objetivos de pesquisa: descrições de fenômenos ou características associadas a uma população-sujeito (o quem, o quê, quando, onde e como de um tópico); estimativas das proporções de uma população que tenha essas características e descoberta de associações entre diferentes variáveis. Um objetivo é a contagem quantitativa de eventos e opiniões, chamada de frequência de resposta. Como estratégia de pesquisa, foi aplicada uma survey, enquanto um interrogatório dos participantes através de um questionário estruturado, destinado a provocar informações específicas dos entrevistados (Malhotra, 2011). Para Creswell (2010), esse tipo de pesquisa apresenta uma descrição quantitativa ou numérica de tendências, atitudes ou opiniões de uma população, estudando-se uma amostra dessa população. A partir dos resultados da amostra, o pesquisador generaliza ou faz afirmações sobre a população.

A população investigada compreendeu docentes que atuam no ensino superior da UFSM, totalizando 1920 docentes, segundo dados disponíveis no Portal da UFSM no mês de setembro de 2020. Para um erro amostral de 6\% e nível de confiança de $95 \%$ a amostra final mínima a ser investigada era de 235 docentes, sendo que ao final da pesquisa obteve-se um total de 247 respondentes. A escolha da população decorreu do fato desse ambiente laboral envolver sobrecarga de trabalho, pressão em termos de produtividade acadêmica, entre outros fatores que acarretam desgaste e desinteresse com o trabalho docente 
(Rahman \& Avan, 2016). No que concerne aos aspectos éticos, o projeto foi aprovado pelo Comitê de Ética em Pesquisa com Seres Humanos da UFSM.

Inicialmente, foram realizados pré-testes com uma pequena amostra de 5 indivíduos para a validação semântica do instrumento. Após os ajustes necessários, iniciou-se a coleta efetiva dos dados, a qual ocorreu entre os meses de setembro e dezembro de 2020. Os sujeitos foram abordados através do Sistema de Questionários da UFSM, o qual é disponibilizado de forma online individualmente para todo os professores. O instrumento foi estruturado com 51 questões, subdivididas em 4 partes, conforme Quadro 1.

Quadro 1 - Resumo do instrumento de coleta de dados.

\begin{tabular}{|c|l|l|l|}
\hline Partes & \multicolumn{1}{|c|}{ Tema } & \multicolumn{1}{|c|}{ Mensuração } & \multicolumn{1}{|c|}{ Referências } \\
\hline I & Perfil & 17 questões abertas e fechadas & Elaborado pelas autoras \\
\hline II & $\begin{array}{l}\text { Situação pessoal e profissional } \\
\text { durante a Pandemia do COVID- } \\
19 .\end{array}$ & 15 questões abertas e fechadas & Elaborado pelas autoras \\
\hline III & $\begin{array}{l}\text { Percepção de Suporte } \\
\text { Organizacional (EPSO) }\end{array}$ & $\begin{array}{l}\text { 9 questões do tipo likert de cinco } \\
\text { pontos (1- Discordo Totalmente à 5- } \\
\text { Concordo Totalmente) }\end{array}$ & $\begin{array}{l}\text { Siqueira e Gomide Júnior } \\
\text { (2008) }\end{array}$ \\
\hline IV & $\begin{array}{l}\text { Engajamento no Trabalho } \\
\text { (EEGT) }\end{array}$ & $\begin{array}{l}10 \text { questões do tipo likert de cinco } \\
\text { pontos (1- Nunca à 5- Sempre). }\end{array}$ & Siqueira et al. (2014) \\
\hline
\end{tabular}

Fonte: Autores (2021).

Os dados foram analisados por meio do software SPSS 20.0 (Statistical Package for the Social Sciences), sendo conduzida a análise a partir da estatística descritiva, análise fatorial exploratória e regressão. A análise fatorial exploratória avalia os agrupamentos e correlações entre as variáveis (Hair, Babin, Money, \& Samouel, 2005), definindo a composição dos fatores de EGT e PSO. Foram aplicados os testes de esfericidade de Barlett e de Kaiser-Meyer-Olkin (KMO). Para manutenção das variáveis usou-se o critério das comunalidades maiores ou iguais a 0,5. O método de componentes principais estimou as cargas fatoriais dos componentes, considerando os critérios dos autovalores maiores que 1,0 e porcentagem da variância explicada para determinar o número de fatores. A confiabilidade dos fatores considerou o Alpha de Cronbach, sendo aceitáveis valores superiores a 0,7 (Hair, Black, Babin, \& Anderson, 2009).

Por fim, foi realizada a análise de regressão, tendo como variável dependente o EGT e como variáveis independentes o PSO e três dummies (sexo, filho, chefia). Para verificar a normalidade do erro, realizou-se o teste de Kolmogorov-Smirnov KS sob a hipótese nula de que a distribuição da série testada é normal. A multicolinearidade dos fatores foi verificada pelo fator de inflação da variância (FIV). O pressuposto da homocedasticidade utilizou o teste de Pesaran-Pesaran, no intuito de verificar se a variância dos resíduos se mantém constante em todo o espectro das variáveis independentes.

\section{Resultados e Discussão}

Esta seção apresenta, inicialmente, uma análise descritiva da amostra, seguida da análise fatorial e discussão sobre os resultados encontrados. Por fim, apresenta-se a análise de regressão e discussões dos dados.

\subsection{Análise descritiva da amostra}

Dentre os respondentes predomina o gênero masculino, com idade entre 37 a 47 anos (38,2\%), sendo a maioria casados (56,5\%). Os entrevistados em geral possuem de 1 a 3 filhos (67,1\%), sendo 35,9\% deles menores que 10 anos. A totalidade dos respondentes reside no Estado do RS, com prevalência da cidade de Santa Maria (85,6\%). Quanto à área de 
formação, predominam as Ciências Sociais Aplicadas (20,8\%) e a pluralidade dos pesquisados possui Grau de Doutorado (68,3\%), com até 10 anos de tempo de serviço $(50,4 \%)$. A maioria dos respondentes não possui cargo/posição de chefia $(72,8 \%)$ e estão vinculados ao Regime de Dedicação Exclusiva (91,9\%), que é considerado flexível pelos entrevistados $(84,1 \%)$. A maioria dos respondentes dispunham de orientandos no decorrer da pesquisa $(81,7 \%)$, tanto de Graduação $(71,3 \%)$, quanto de Pós-Graduação (57,3\%), orientando de 1 a 4 estudantes.

Em relação a Pandemia da COVID-19, 31,3\% dos docentes fazem parte do grupo de risco e a maioria $(53,7 \%)$ declarou viver em um nível médio de isolamento social - visitando apenas familiares esporadicamente -, desde o início da pandemia $(48,4 \%)$. Em relação à dinâmica familiar, a maior parte dos docentes $(27,64 \%)$ reside com três pessoas e $34,5 \%$ afirmou fornecer assistência ou cuidado especial a pessoa com mais de 65 anos. A maioria está atuando em regime de homeoffice $(94,7 \%)$ desde o início da pandemia $(94,8 \%)$ e trabalha de 5 a 9 horas por dia $(52,4 \%)$. Em contrapartida, essa dedicação reduz em relação às horas diárias dedicadas à família, que representa de 1 a 4 horas $(48,8 \%)$.

No tocante a forma como os pesquisados passaram a ministrar as aulas, a maioria adotou o sistema de aulas virtuais (50\%), envolvendo mais de 20 horas por semana para preparar o material didático (34,8\%). Em relação a atuação dos professores na pós-graduação, a maioria não ministrou disciplinas desde o início da pandemia $(61,78 \%)$. Outro dado relevante é que $78,5 \%$ dos docentes afirmam que suas chefias e/ou instituição proporcionaram espaços para discussões e reflexões sobre o andamento das atividades acadêmicas, classificando como suficiente as orientações recebidas durante este período $(61,8 \%)$.

\subsection{Análise fatorial da percepção de engajamento no trabalho}

O indivíduo ao se sentir engajado tende a ver o trabalho como algo estimulante, para o qual realmente deseja dedicar tempo e esforço, percebendo seu significado (Bakker, Albrecht, \& Leiter, 2011). Neste sentido, colaboradores engajados tendem a uma maior probabilidade de se envolver em novos desafios e descobertas, apresentando melhor desempenho, uma vez que, estão mais dispostos a encarar com proatividade suas atividades (Caesens \& Stinglhamber, 2014; Karatepe \& Aga, 2016; Tan et al., 2020). O EGT está atrelado a uma concepção mais complexa e ampla sobre o relacionamento do indivíduo com o trabalho, de modo que o foco reside no trabalho em si, podendo ser influenciado por emoções e comportamentos compartilhados através das interações entre membros da organização (Santos, Costa, Sá Brito, \& Salles, 2019).

Em relação ao EGT, 45\% dos docentes acreditam que muitas vezes ficam cheios de energia quando estão trabalhando, mantêm o pensamento voltado apenas para as suas tarefas $(56,5 \%)$ e sentem-se revigorados (38,6\%). Percebeu-se, que muitas vezes, os docentes dizem-se estar concentrados em suas tarefas (56,5\%) e dispostos (48,8\%), estando, às vezes, distantes de seus problemas pessoais $(39,0 \%)$. Além disso, os entrevistados, muitas vezes, estão focados no que estão fazendo (54,9\%), fortalecidos $(37,8 \%)$, renovados $(36,6 \%)$ e tomados por suas tarefas $(45,5 \%)$, o que destaca uma orientação positiva em relação ao EGT. Tais dados corroboram com os achados de Schaufeli, Dijkstra e Vazquez (2013) ao sinalizar que mesmo que os funcionários enfrentem algum tipo de adversidade podem apresentar alto engajamento.

A análise fatorial (Tabela 1) estabeleceu a formação dos fatores da dimensão EGT, sendo excluídas a variáveis "Tomado por minhas tarefas" $(0,361)$ e "Distante dos meus problemas pessoais" $(0,377)$ por apresentarem baixa comunalidade extraída. A medida KMO apresentou o valor de 0,863, o teste de esfericidade de Bartlett foi significativo ( $\operatorname{sig}<0,001$ ), confirmando a fatorabilidade dos dados. Quanto à variância, verifica-se que os dois fatores que apresentaram autovalores superiores a um, em conjunto, explicam $76,46 \%$ de toda a variância. 
Tabela 1 - Composição da dimensão Engajamento no Trabalho: Alpha de Crombach, Média e Variância dos fatores, e Média e Carga Fatorial das Variáveis.

\begin{tabular}{|c|c|c|c|}
\hline Variável & Média & Carga & Variância \\
\hline Fator 1: Estado de Espírito (Alpha de Crombach: 0,930) & 3,42 & - & \multirow{6}{*}{$47,85 \%$} \\
\hline Cheio de energia & 3,57 & 0,805 & \\
\hline Revigorado & 3,22 & 0,904 & \\
\hline Disposto & 3,63 & 0,762 & \\
\hline Fortalecido & 3,39 & 0,895 & \\
\hline Renovado & 3,28 & 0,905 & \\
\hline Fator 2: Foco nas tarefas (Alpha de Crombach: 0,795) & 3,91 & - & \multirow{4}{*}{$28,61 \%$} \\
\hline Com o pensamento voltado apenas para as minhas tarefas & 3,82 & 0,768 & \\
\hline Concentrado em minhas tarefas & 3,94 & 0,836 & \\
\hline Focado no que estou fazendo & 3,98 & 0,839 & \\
\hline
\end{tabular}

Fonte: Resultados da pesquisa (2021).

Destaca-se que os Fatores 1 e 2 representam confiabilidade satisfatória conforme o Alpha de Cronbach de 0,930 e 0,795, respectivamente. Ainda, a média de 3,42 para o Fator 1 sugere um elevado grau de EGT dos docentes, o que é refletido na disposição e energia dos colaboradores. Por outro lado, a média de 3,91 para o Fator 2 destaca um elevado grau de frequência relacionado ao EGT, especialmente na variável "focado no que estou fazendo".

\subsection{Análise fatorial da percepção de suporte organizacional}

As crenças e as expectativas do indivíduo acerca da retribuição e do reconhecimento dado pela organização ao seu trabalho são fatores imbricados a PSO (Eisenberger, Huntington, Hutchison, \& Sowa, 1986). Tal compreensão desperta a preocupação das organizações em oferecer recursos que permitam amparar os indivíduos no desenvolvimento de suas atividades laborais da melhor forma possível, no intuito de obter resultados benéficos ao trabalho, incluindo maior comprometimento organizacional (Najeemdeen et al., 2018) e menor absenteísmo e rotatividade (Kurtessis, Eisenberger, Ford, Buffardi, Stewart, \& Adis, 2017; Siqueira \& Gomide Júnior, 2008).

Trata-se da percepção do trabalhador sobre características e condições de trabalho, estando atrelado à frequência, intensidade e sinceridade dos elogios e recompensas que a organização dirige aos seus membros (Siqueira \& Gomide Júnior, 2008). De acordo com Eisenberger e Stinglhamber (2011) e Siqueira e Gomide Júnior (2008), envolve elementos como comprometimento organizacional, satisfação no trabalho, desempenho e produtividade.

$\mathrm{Na}$ análise da PSO, observou que os docentes discordam sobre a instituição ignorar qualquer reclamação da sua parte $(39,4 \%)$ e em relação a não considerar seus interesses quando toma decisões que os afetam $(37,8 \%)$, demonstrando uma maior PSO. Os entrevistados concordam sobre as premissas de que obtêm ajuda da instituição se tiverem um problema (56,1\%) e sobre a mesma realmente preocupar-se com seu bem-estar $(43,5 \%)$.

A maior parte dos docentes, não concorda nem discorda sobre a instituição estar disposta a ampliar suas instalações para os ajudar a utilizar as melhores habilidades no desempenho do trabalho $(42,7 \%)$ ou sobre estar pronta a ajudar quando o colaborador precisar de um favor especial $(43,1 \%)$. Além disso, concordam quanto a percepção de que a instituição se preocupa com sua satisfação no trabalho $(45,5 \%)$ e em fazer com que seu trabalho seja o mais interessante possível (39\%). Quanto a análise fatorial (Tabela 2), a formação dos fatores de PSO baseou-se nas orientações de Siqueira e Gomide Júnior (2008, p. 289) obtendo-se o escore médio da EPSO pela "soma dos valores indicados pelo respondente dentro dos parênteses e dividindo-se esta somatória por nove”. Assim, as variáveis com formulação invertida (sinalizadas com **) tiveram a sua 
pontuação atribuída invertida, antes de se proceder ao cômputo do escore médio.

O primeiro ponto a destacar é que as variáveis invertidas resultaram na indicação de que há suporte organizacional na instituição. Os valores da Tabela 2 para essas questões já foram invertidos, o que significa que, em média, os respondentes discordam do conteúdo dessas questões. Pode-se verificar que a média geral do fator $(3,59)$ dá subsídios de que há suporte organizacional na empresa.

Tabela 2 - Composição da dimensão Percepção de Suporte Organizacional: Alpha de Crombach, Média e Variância dos fatores, e Média e Carga Fatorial das Variáveis.

\begin{tabular}{|c|c|c|c|}
\hline Variável & Média & Carga & Variância \\
\hline Fator 1: Suporte Organizacional (Alpha de Crombach: 0,915) & 3,59 & - & \multirow{10}{*}{$60,14 \%$} \\
\hline Esta instituição ignoraria qualquer reclamação de minha parte** & 3,94 & 0,893 & \\
\hline $\begin{array}{l}\text { Esta instituição não considera meus interesses quando toma decisões que me } \\
\text { afetam** }\end{array}$ & 3,67 & 0,863 & \\
\hline É possível obter ajuda desta instituição quando tenho um problema & 3,86 & 0,828 & \\
\hline Esta instituição realmente preocupa-se com meu bem-estar & 3,59 & 0,815 & \\
\hline $\begin{array}{l}\text { Esta instituição estaria disposta a ampliar suas instalações para me ajudar a } \\
\text { utilizar minhas melhores habilidades no desempenho do meu trabalho }\end{array}$ & 3,07 & 0,792 & \\
\hline Esta instituição está pronta a ajudar-me quando eu precisar de um favor especial & 3,19 & 0,752 & \\
\hline Esta instituição preocupa-se com minha satisfação no trabalho & 3,48 & 0,741 & \\
\hline Esta instituição preocupa-se mais com seus lucros do que comigo** & 4,02 & 0,681 & \\
\hline $\begin{array}{l}\text { Esta instituição tenta fazer com que meu trabalho seja o mais interessante } \\
\text { possível }\end{array}$ & 3,45 & 0,562 & \\
\hline
\end{tabular}

Fonte: Resultados da pesquisa (2021).

Ou seja, há a percepção de que a instituição não ignoraria ou desconsideraria reclamações ou interesses nas decisões que me afetam, bem como está disposta a ajudar em situações que o servidor possui problemas e preocupa-se com seu bemestar. Destaca-se também que o fator apresenta confiabilidade satisfatória (Alpha $=0,915$ ). De acordo com a interpretação do Instrumento de Suporte de Siqueira e Gomide Júnior (2008), os resultados médios sugerem que os docentes percebem de forma duvidosa a preocupação da instituição, com o seu bem-estar.

\subsection{Análise de Regressão para os Fatores de Suporte Organizacional como Preditores do Engajamento no Trabalho}

Através de um modelo de efeitos principais, Mahon, Taylor e Boyatzis (2014) testaram se o PSO se associa positivamente ao EGT, com resultados que confirmaram tal hipótese. Tal raciocínio é consistente com o estudo de Saks e Rotman (2006), que identificaram a influência positiva do PSO no engajamento dos funcionários em termos de empoderamento da organização. Essa relação foi observada no desempenho de professores durante a Pandemia da COVID-19, os quais conseguiram envolver os alunos através de atividades criativas, pois obtinham os recursos necessários, ou seja, infraestrutura e alto grau de alfabetização online, suporte do governo formal e de administradores (Bubb \& Jones, 2020).

De acordo com os resultados Shantz, Alfes e Latham (2014) um baixo nível de engajamento no trabalho só é associado a um maior nível de intenções de rotatividade quando funcionários não percebem que são apoiados por sua organização, ou seja, o PSO compensa níveis relativamente baixos de engajamento no trabalho. Em síntese, à medida que um indivíduo se sentir valorizado, isto é, perceber o suporte, maior será o EGT (Imran, Elahi, Abid, Ashfaq, \& Ilyas, 2020; Kose, 2016, Ahmed e Nawaz, 2015).

No presente estudo, para investigar a influência da PSO sobre o EGT dos docentes que atuam no ensino superior da 
UFSM, realizou-se uma análise de regressão linear, onde o engajamento, estado de espírito e foco nas tarefas representam as variáveis dependente, e o fator PSO, bem como as variáveis binárias sexo, filhos e chefia representam as variáveis independentes. Foram construídos três modelos de regressão, através do método Enter (Tabela 3).

Observa-se que o fator PSO é significativo e exerce influência positiva sobre as variáveis dependentes Engajamento e Estado de Espírito, indo ao encontro dos achados de Eisenberger e Stinglhamber (2011), ao sugerir que, ao reforçar o interesse intrínseco dos funcionários em sua tarefa, o PSO aumentaria o EGT.

Tabela 3 - Coeficientes dos modelos de regressão.

\begin{tabular}{|c|c|c|c|c|c|c|c|c|}
\hline \multirow{2}{*}{ Modelo } & \multirow{2}{*}{ Variável Dependente } & \multirow{2}{*}{$\begin{array}{c}\text { Variável } \\
\text { Independente }\end{array}$} & \multicolumn{2}{|c|}{ Coeficiente } & \multirow{2}{*}{$\mathbf{R}^{2}$} & \multicolumn{2}{|c|}{$\mathbf{F}$} & \multirow{2}{*}{ VIF } \\
\hline & & & Valor & Sig. & & Valor & Sig. & \\
\hline \multirow{4}{*}{1} & \multirow{4}{*}{ Engajamento } & $\begin{array}{l}\text { Suporte } \\
\text { Organizacional }\end{array}$ & 0,347 & 0,000 & \multirow{4}{*}{0,131} & \multirow{4}{*}{9,097} & \multirow{4}{*}{0,000} & 1,016 \\
\hline & & Dummy Sexo & 0,055 & 0,364 & & & & 1,008 \\
\hline & & Dummy Filhos & $-0,044$ & 0,469 & & & & 1,016 \\
\hline & & Dummy Chefia & $-0,054$ & 0,375 & & & & 1,011 \\
\hline \multirow{4}{*}{2} & \multirow{4}{*}{ Fator 1 - Estado de Espírito } & $\begin{array}{l}\text { Suporte } \\
\text { Organizacional }\end{array}$ & 0,463 & 0,000 & \multirow{4}{*}{0,217} & \multirow{4}{*}{16,726} & \multirow{4}{*}{0,000} & 1,016 \\
\hline & & Dummy Sexo & 0,010 & 0,858 & & & & 1,008 \\
\hline & & Dummy filhos & $-0,088$ & 0,129 & & & & 1,016 \\
\hline & & Dummy Chefia & $-0,009$ & 0,871 & & & & 1,011 \\
\hline \multirow{4}{*}{3} & \multirow{4}{*}{ Fator 2 - Foco nas tarefas } & $\begin{array}{l}\text { Suporte } \\
\text { Organizacional }\end{array}$ & 0,091 & 0,158 & \multirow{4}{*}{0,029} & \multirow{4}{*}{1,785} & \multirow{4}{*}{0,133} & 1,016 \\
\hline & & Dummy Sexo & 0,094 & 0,141 & & & & 1,008 \\
\hline & & Dummy Filhos & 0,025 & 0,692 & & & & 1,016 \\
\hline & & Dummy Chefia & $-0,093$ & 0,147 & & & & 1,011 \\
\hline
\end{tabular}

Fonte: Resultados da pesquisa (2021).

Esses resultados corroboram Mahon et al. (2014), os quais confirmaram que o PSO se associa positivamente ao engajamento organizacional, assim como Saks e Rotman (2006) e Ahmed e Nawaz (2015) que identificam a influência positiva do PSO no engajamento dos funcionários. Os resultados também vão ao encontro dos estudos que utilizaram o Modelo JD-R para demonstrar que os recursos do trabalho (PSO) gerados pelo humor do líder (Tan et al., 2020) ou pelo suporte social (Park, Lim, Kim, \& Kang, 2020) estão relacionados ao EGT. No mesmo sentido, Chanana (2020) afirma que para manter o alto engajamento entre os funcionários, os gerentes devem fornecer feedback e orientação eficaz aos seus subordinados.

No Modelo 3, nenhuma das constantes é significativa, o que significa que nenhuma delas exerce influência sobre o Fator 2 - foco nas tarefas. Isso pode ser explicado devido ao contexto de aplicação dos questionários em momento da Pandemia da COVID-19, com questionários que não foram atualizados para se adequarem a tal contexto. Em relação aos pressupostos de normalidade (teste KS), todos os Modelos apresentam a significância. Quanto aos índices VIF, os modelos válidos também apresentam valores satisfatórios ao pressuposto $(\mathrm{VIF}=1,0)$, não registrando problemas de multicolineariedade. O teste Pesarán-Pesarán indicou que os Modelos 1 e 2 atendem o pressuposto da homoscedasticidade (Sig. 0,4), aceitando a hipótese nula de que os erros são homocedásticos.

\section{Conclusão}

Este estudo teve como objetivo identificar a influência do suporte organizacional sobre o engajamento no trabalho durante a Pandemia da COVID-19. A análise fatorial resultou em dois fatores para a dimensão EGT, os quais representam $76,46 \%$ da variância total acumulada, tendo sua consistência interna assegurada pelo teste de confiabilidade. A média do Fator 1 - Estado de Espírito $(3,42)$ sugere um elevado grau de EGT dos docentes, refletindo-se principalmente nas variáveis de 
disposição e energia dos colaboradores. Para o Fator 2 - Foco nas Tarefas observa-se que um alto grau de frequência relacionado ao EGT $(3,91)$, sendo mais representativo em relação ao foco no que o docente está fazendo.

Esses resultados demostram alto grau de EGT durante o período de início de pandemia, mesmo diante de uma alta carga horária de trabalho. Tal achado corrobora com Bakker e Demerouti (2008), em relação a possibilidade de o engajamento criar uma espiral de ganhos positivos, à medida que os funcionários são capazes de criar seus próprios recursos.

Outra questão que pode ter influenciado o alto grau de engajamento é o fato da maioria dos professores estarem realizando trabalho remoto, o que remete ao estudo de Znidarsic e Bernic (2021), os quais confirmaram que a percepção de PSO por indivíduos no equilíbrio entre trabalho e vida familiar tem uma influência positiva no EGT. Os autores evidenciaram que o número de horas trabalhadas por semana não tem impacto significativo no equilíbrio trabalho-família, o que compactua com o presente estudo, pois embora os docentes relatem que dispensam mais horas ao trabalho do que à família, isso não afetou seu engajamento.

Ressalta-se ainda, que a média de 3,59 para o SO (Siqueira \& Gomide Júnior, 2008) sinaliza que os docentes percebem falta de preocupação da empresa com o seu bem-estar, entretanto, considera-se que as variáveis que apresentam maior média são invertidas e que a variável que apresentou maior média refere-se a 'lucros', elemento que não faz parte do universo de Universidades públicas. Pode-se inferir que os docentes percebem que a instituição está preocupada em promover o seu bem-estar, ainda que em alguns pontos percebam falta de preocupação em relação ao seu bem-estar. Torna-se relevante a revisão e desenvolvimento de políticas internas sobre o suporte organizacional que integrem a experiência de trabalho dos funcionários, buscando fornecer rearranjos que auxiliem o bem-estar, considerando a importância do suporte organizacional, conforme aponta o estudo de Mihalache e Mihalache (2021).

Foram propostos três modelos de regressão que têm como variável dependente os fatores de EGT (e o próprio EGT) e como variável independente o fator de PSO. Os resultados demonstraram a influência de PSO sobre EGT e Estado de Espírito. Esses resultados geram insights para o avanço da literatura em um contexto de pandemia, e atenta para a necessidade de se investigar outros aspectos relativos ao ambiente laboral que interferem no EGT.

Considerando que a Pandemia da COVID-19 impactou a dinâmica de trabalho dos docentes, argumenta-se que para estarem engajados devem perceber o suporte organizacional. Assim, sugere-se verificar e considerar a importância do suporte organizacional nas políticas e regulamentos durante a COVID-19, reconhecendo que as modificações na dinâmica de trabalho envolvem não só os riscos de controle administrativo e proteção individual, mas questões sociais do trabalho, como a própria virtualização das relações. A influência do suporte organizacional no engajamento dos docentes pode apoiar-se na realização de feedback, comunicação adequada, suporte tecnológico, webinars, tanto de treinamento como de atividades para o bem-estar, mantendo a interação e motivação dos funcionários.

Estudos futuros devem ser realizados a fim de melhor explorar o comportamento do fator de PSO em contextos de trabalho remoto, já que este indicou resultados válidos, o que sugere a necessidade de usar outras variáveis que possam influenciar o EGT nesta dinâmica de trabalho. Diante disso, reaplicações com amostras variadas e outros contextos de trabalho remoto poderão contribuir para que se possa chegar a um consenso relativo a esses dois instrumentos. Enquanto limitações, salienta-se que as escalas utilizadas, tanto de EGT quanto de PSO, não haviam sido utilizadas nem testadas em um contexto de Pandemia.

\section{Referências}

Ahmed, I., \& Nawaz, M. M. (2015). Antecedents and outcomes of perceived organizational support: a literature survey approach. Journal of Management Development, 34(7), 867-880.http://doi:10.1108/jmd-09-2013-0115

Adedoyin, O. B., \& Soykan, E. (2020). Covid-19 pandemic and online learning: the challenges and opportunities. Interactive Learning Environments, 1-13. Advanced online publication. https://doi.org/10.1080/10494820.2020.1813180 
Anderson, D., \& Kelliher, C. (2020). Enforced remote working and the work-life interface during lockdown. Gender in Management: An International Journal, 35(7/8), 677-683. https://doi.org/10.1108/GM-07-2020-0224

Bakker, A. B., Albrecht, S. L., \& Leiter, M. P. (2011). Key questions regarding work engagement. European journal of work and organizational psychology, 20(1), 4-28. https://doi.org/10.1080/1359432X.2010.485352

Bakker, A. B., \& Demerouti, E. (2008). Towards a model of work engagement. Career development international, 13(3), $209-223$. https://doi.org/10.1108/13620430810870476

Bao, W. (2020). COVID-19 and online teaching in higher education: A case study of Peking University. Human Behavior and Emerging Technologies, 2(2), 113-115. https://doi.org/10.1002/hbe2.191

Bubb, S., \& Jones, M. A. (2020). Learning from the COVID-19 home-schooling experience: Listening to pupils, parents/carers and teachers. Improving schools, 23(3), 209-222. https://doi.org/10.1177/1365480220958797

Caesens, G., \& Stinglhamber, F. (2014). The relationship between perceived organizational support and work engagement: The role of self-efficacy and its outcomes. European Review of Applied Psychology, 64 (5), 259-267. https://doi.org/10.1016/j.erap.2014.08.002

Cardullo, V., Wang, C. H., Burton, M., \& Dong, J. (2021). K-12 teachers' remote teaching self-efficacy during the pandemic. Journal of research in innovative teaching \& learning, 14(1), 32-45. https://doi.org/10.1108/JRIT-10-2020-0055

Chanana, N. (2020). Employee engagement practices during COVID-19 lockdown. Journal of Public Affairs, e2508, 1-8. https://doi.org/10.1002/pa.2508

Diab-Bahman, R., \& Al-Enzi, A. (2020). The impact of COVID-19 pandemic on conventional work settings. International Journal of Sociology and Social Policy, 40(9/10), 909-927. https://doi.org/10.1108/ISSSP-07-2020-0262

Donaldson, J. P. (2020). Building a digitally enhanced community of practice. Information and Learning Sciences, 121(5/6), 241-250. https://doi.org/10.1108/ILS-04-2020-0066

Eisenberger, R., Huntington, R., Hutchison, S., \& Sowa, D. (1986). Perceived organizational support. Journal of Applied psychology, 71(3), 500. https://doi.org/10.1037/0021-9010.71.3.500

Eisenberger, R., \& Stinglhamber, F. (2011). Perceived organizational support: Fostering enthusiastic and productive employees. Washington, DC: American Psychological Association. https://doi.org/10.1037/12318-000

Erum, H., Abid, G., \& Contreras, F. (2020). The calling of employees and work engagement: the role of flourishing at work. Business, management and education, 18(1), 14-32. https://doi.org/10.3846/bme.2020.11430

Garg, N., Murphy, W., \& Singh, P. (2021). Reverse mentoring, job crafting and work-outcomes: the mediating role of work engagement. Career Development International, 26(2), 290-308. https://doi.org/10.1108/CDI-09-2020-0233

Gupta, M., Shaheen, M., \& Reddy, P. K. (2017). Impact of psychological capital on organizational citizenship behavior. Journal of Management Development, 36(7), 973-983. http://doi:10.1108/jmd-06-2016-0084

Hair Jr, J. F., Babin, B., Money, A. H., \& Samouel, P. (2005). Fundamentos de métodos de pesquisa em administração. Porto Alegre: Bookman.

Hair, J. F., Black, W. C., Babin, B. J., Anderson, R. E., \& Tatham, R. L. (2009). Análise multivariada de dados. (6a ed.). Porto Alegre: Bookman.

Hoang, A. D. (2020). Pandemic and teacher retention: empirical evidence from expat teachers in Southeast Asia during COVID-19. International Journal of Sociology and Social Policy, 9/10(40), 1141-1166. https://doi.org/10.1108/ISSP-07-2020-0269

Imran, M. Y., Elahi, N. S., Abid, G., Ashfaq, F., \& Ilyas, S. (2020). Impact of perceived organizational support on work engagement: Mediating mechanism of thriving and flourishing. Journal of Open Innovation: Technology, Market, and Complexity, 6(3), 82. https://doi.org/10.3390/joitmc6030082

Kamble, A., Gauba, R., Desai, S., \& Golhar, D. (2021). Learners' Perception of the Transition to Instructor-Led Online Learning Environments: Facilitators and Barriers During the COVID-19 Pandemic. International Review of Research in Open and Distributed Learning, 22(1), 199-215. https://doi.org/10.19173/irrodl.v22i1.4971

Karatepe, O. M., \& Aga, M. (2016). The effects of organization mission fulfillment and perceived organizational support on job performance: The mediating role of work engagement. International Journal of Bank Marketing, 34(3), 368-387. https://doi.org/10.1108/IJBM-12-2014-0171

Kashyap, V., Nakra, N., \& Arora, R. (2021). Do “decent work” dimensions lead to work engagement? Empirical evidence from higher education institutions in India. European Journal of Training and Development, Vol. ahead-of-print No. ahead-of-print. https://doi.org/10.1108/EJTD-08-2020-0127

Kose, M. A. (2016). Comments on "Pareto weights as wedges in two-country models" by D. Backus, C. Coleman, A. Ferriere and S. Lyon. Journal of Economic Dynamics and Control, 72, 111-114. https://doi.org/10.1016/j.jedc.2016.05.001

Kurtessis, J. N., Eisenberger, R., Ford, M. T., Buffardi, L. C., Stewart, K. A., \& Adis, C. S. (2017). Perceived organizational support: A meta-analytic evaluation of organizational support theory. Journal of management, 43(6), 1854-1884. https://doi.org/10.1177/0149206315575554

Mahon, E. G., Taylor, S. N., \& Boyatzis, R. E. (2014). Antecedents of organizational engagement: exploring vision, mood and perceived organizational support with emotional intelligence as a moderator. Frontiers in psychology, 5(1322), 1-11. https://doi.org/10.3389/fpsyg.2014.01322

Mihalache, M., Mihalache, O. R. (2021). How workplace support for the COVID-19 pandemic and personality traits affect changes in employees' affective commitment to the organization and job-related well-being. Human Resource Management, 1-20. http://doi:10.1002/hrm.22082 
Najeemdeen, I. S., Abidemi, B. T., Rahmat, F. D., \& Bulus, B. D. (2018). Perceived organizational culture and perceived organizational support on work engagement. Academic Journal of Economic Studies, 4(3), 199-208. http://www.discoveryjournals.org/discovery/current_issue/v54/n275/A1.pdf?

Park, Y., Lim, D. H., Kim, W., \& Kang, H. (2020). Organizational Support and Adaptive Performance: The Revolving Structural Relationships between Job Crafting, Work Engagement, and Adaptive Performance. Sustainability, 12(12), 4872. https://doi.org/10.3390/su12124872

Rahman, M., \& Avan, Y. R. (2016). Teaching workload and performance: An empirical analysis on selected private universities of Bangladesh. European Journal of Social Sciences Studies, 1(1), 71-83. http://dx.doi.org/10.46827/ejsss.v0i0.5

Rapanta, C., Botturi, L., Goodyear, P., Guàrdia, L., \& Koole, M. (2020). Online university teaching during and after the Covid-19 crisis: Refocusing teacher presence and learning activity. Postdigital Science and Education, 2(3), 923-945. https://doi.org/10.1007/s42438-020-00155-y

Reimers, F., Schleicher, A., Saavedra, J., \& Tuominen, S. (2020). Supporting the continuation of teaching and learning during the COVID-19 Pandemic. Oecd, 1(1), 1-38. Recuperado de https://www.oecd.org/education/Supporting-the-continuation-of-teaching-and-learning-during-the-COVID-19-pandemic.pdf

Rodrigues, M., Franco, M., \& Silva, R. (2020). COVID-19 and disruption in management and education academics: Bibliometric mapping and analysis. Sustainability, 12(18), 7362. https://doi.org/10.3390/su12187362

Santos, S. D. S. C., Costa, I. D. S. A., Sá Brito, J. A., \& Salles, D. M. R. (2019). Percepções de engajamento no trabalho sob a ótica do modelo JD-R: estudo em um Centro Federal de Educação Tecnológica no Rio de Janeiro. Revista Organizações em Contexto, 15(30), 121-158. https://doi.org/10.15603/19828756/roc.v15n30p121-158

Saks, A. M., \& Rotman, J. L. (2006). Antecedents and consequences of employee engagement. Journal of Managerial Psychology, 21(7), 600-619. https://doi.org/10.1108/02683940610690169

Sarassa, J. B., \& Peréz, E. R. (2019). Empleabilidad y trabajo del profesorado universitario: una revision del campo. Athenea digital: revista de pensamiento e investigación social, 19(3), e2140. https://doi.org/10.5565/rev/athenea.2140

Schantz, A., Alfes, K., Lathan, G. P. (2014) The buffering effect of perceived organizational support on the relationship between work engagement and behavioral outcomes. Human Resource Management. http://doi:10.1002/hrm.21653

Schaufeli, W. B., Dijkstra, P., \& Vazquez, A. C. (2013). Engajamento no trabalho. São Paulo: Casa do Psicólogo.

Shi, X. (C.)., \& Gordon, S. (2020). Organizational support versus supervisor support: The impact on hospitality managers psychological contract and work engagement International. Journal of Hospitality Management, 87. https://doi.org/10.1016/j.ijhm.2019.102374

Siqueira, M. M. M., \& Gomide Júnior, S. Suporte no trabalho. In M. M. M. Siqueira. (Ed.). (2008) Medidas do comportamento organizacional: ferramentas de diagnóstico e de gestão. Porto Alegre: Artmed Editora, 2008, 277-288.

SIQUEIRA, M. M. M.; MARTINS, M. C. F.; ORENGO, V.; SOUZA, W. Engajamento no trabalho. In: SIQUEIRA, M.M.M (Org) (2014). Novas Medidas do Comportamento Organizacional. Ferramentas de Diagnóstico e Gestão. Porto Alegre: Artmed, p. 147-154.

Tan, L., Wang, Y., Qian, W., \& Lu, H. (2020). Leader humor and employee job crafting: the role of employee-perceived organizational support and work engagement. Frontiers in Psychology, 11, 2592. https://doi.org/10.3389/fpsyg.2020.499849

Tavares, F., Santos, E., Diogo, A., \& Ratten, V. (2020). Teleworking in Portuguese communities during the COVID-19 pandemic. Journal of Enterprising Communities: People and Places in the Global Economy, 15(3), 334-349. https://doi.org/10.1108/JEC-06-2020-0113

UFSM. (2020a). Universidade Federal de Santa Maria. Instrução Normativa $n^{o}$ 02/2020. Santa Maria. Recuperado de https://drive.google.com/file/d/1kY-nMuEkBybVZN1y1S3wcXbXi5gRD-A/view

(2020b). Universidade Federal de Santa Maria. Instrução Normativa $n^{o}$ 02/2020. Santa Maria. Recuperado de https://www.ufsm.br/proreitorias/proplan/resolucao-n-024-2020

Znidarsic, J., \& Bernik, B. Impact of work-family balance results on employee work engagement within the organization: The case of Slovenia. Impact of work-family balance results on employee work engagement within the organization: The case of Slovenia. PLoS ONE 16(1): e0245078. https://doi.org/10.1371/journal.pone.0245078

Zhou, L., Wu, S., Zhou, M., \& Li, F. (2020). 'School's Out, But Class' On', The Largest Online Education in the World Today: Taking China's Practical Exploration During The COVID-19 Epidemic Prevention and Control As an Example. Best Evid Chin Edu, 4(2), 501519. http://dx.doi.org/10.2139/ssrn.3555520 